\title{
Paper
}

\section{Nonlinear portfolio model and its rebalancing strategy}

\author{
Satoshi Inose $^{1 a)}$, Tomoya Suzuki ${ }^{2}$, and Kazuo Yamanaka ${ }^{2}$ \\ ${ }^{1}$ Graduate School of Science and Engineering, Ibaraki University \\ 4-12-1 Nakanarusawa-cho, Hitachi-shi, Ibaraki, 316-8511, Japan \\ ${ }^{2}$ College of Engineering, Ibaraki University \\ 4-12-1 Nakanarusawa-cho, Hitachi-shi, Ibaraki, 316-8511, Japan \\ a)12nd202x@hcs.ibaraki.ac.jp
}

Received January 10, 2013; Published October 1, 2013

\begin{abstract}
A nonlinear portfolio model was formulated by applying a nonlinear prediction method and its prediction error to the Markowitz mean-variance portfolio model. Also, the Sharpe ratio, which is a typical evaluation function of portfolio optimization, was modified to adopt stock-trading commissions and the trading-unit system, which are inevitable for portfolio rebalancing in real investment. Then, we discussed the best rebalancing frequency from the viewpoint of the trade-off between prediction accuracy and rebalancing costs. By investment simulations based on real stock data, we confirmed that shorter-term rebalancing is more effective even if we are required to pay higher commissions because short-term nonlinear prediction works better to estimate future return rates and to reduce investment risks.
\end{abstract}

Key Words: nonlinear prediction model, portfolio theory, financial engineering

\section{Introduction}

The Markowitz mean-variance portfolio model [1] has been used to optimize stock allocation rates by maximizing the future return rate of a portfolio set and minimizing the risk of it. According to this model, the future return rate is estimated as the most probable value, and the risk is estimated as a possible error of the estimated future return rate. For the estimation, we have to obtain the probability distribution of the future return rate. However, because this probability distribution is completely unknown, the previous model substitutes the mean value and the standard deviation of historical return rates as the future return rate and the risk.

From the viewpoint of time-series prediction, this estimation of the future return rate might be too simple to predict real financial markets because the mean value of historical data corresponds to simply averaging the past data. If financial markets are efficient and unpredictable as Fama mentioned [2], it is meaningless to improve prediction models. However, various inefficiencies in real financial markets have been reported through many empirical analyses. Especially, market anomalies can be confirmed in daily data and are reduced as the time scale is larger, like weekly data or monthly data [3].

To model such properties of real markets, some mathematical models have been proposed. As prediction models of future return rates, the AR model, the ARMA model, etc. have been applied [4]. 
However, this prediction accuracy is not good enough. For this reason, some fundamental factors such as macroeconomic and/or financial indexes have been applied to compose a multi-factor model. Especially, the Black-Litterman model is well-known recently which uses not only historical return rates but also investor's subjective view to estimate the future return rate [5]. However, because the propose of our study is to modify the Markowitz mean-variance portfolio model, we try to make a portfolio only by using the historical return rates. Therefore, we discuss how to apply more advanced prediction models to the original portfolio model. However, if we change how to expect future return rates, we have to reconsider the risk defined by the original model. Here, if we regard the risk as the probability that the expected future will not be realized, we could estimate the risk by following historical prediction errors. To confirm the validity of our method, we perform investment simulations based on real stock data.

Moreover, we discuss the rebalancing strategy to keep a portfolio fresh from rapid change of market trends. In real investment, we have to pay stock-trading commissions to rebalance a portfolio, and we cannot trade the odd amount of shares because the minimum trading amount (the trading unit) of each stock is decided like 100 shares, 1000 shares, etc. However, these details are omitted in the Sharpe ratio [6], which is a typical evaluation function of portfolio optimization. Therefore, we have to modify the Sharpe ratio to adopt these details, which are essential factors in the present study because stock-trading commissions influence the rebalancing strategy and the trading-unit system changes the class of portfolio optimization into the integer optimization. The accuracy of this integer optimization depends on the amount of trading assets, and therefore we examine this dependence by numerical simulations. In addition, the rebalancing frequency has the trade-off between prediction accuracy and rebalancing costs. If the rebalancing frequency is increased, the rebalanced portfolio can follow the rapid change of market trends, but we have to pay more commissions for each portfolio rebalancing. On the other hand, if the rebalancing frequency is decreased, we can reduce commissions, but the portfolio can not follow market trends. Therefore, we examine the best rebalancing frequency by investment simulations based on the modified Sharpe ratio considering stock-trading commissions and the trading-unit system as rules of real investment.

\section{The Markowitz mean-variance portfolio model}

In this section, we do not yet consider stock-trading commissions and the trading-unit system to introduce the original Markowitz portfolio model. First, we denote $x_{i}(t)$ as the price of $i$ th stock $(i=1,2, \cdots, N)$ at the time of $t$, and the return rate for $s$ period $r_{s, i}(t)$ is given by

$$
r_{s, i}(t)=\frac{x_{i}(t)-x_{i}(t-s)}{x_{i}(t-s)} .
$$

Next, we denote $P^{(k)}$ as the probability that $r_{s, i}(t+s)$ becomes $r_{s, i}^{(k)}(t+s)$ at the future time of $t+s$, where $\sum_{k=1}^{K} P^{(k)}=1$. Thus, the expected return rate $\tilde{r}_{s, i}(t+s)$ and the variance $\tilde{\sigma}_{s, i}(t+s)$ are respectively given by

$$
\begin{aligned}
& \tilde{r}_{s, i}(t+s)=\sum_{k=1}^{K} P^{(k)} r_{s, i}^{(k)}(t+s), \\
& \tilde{\sigma}_{s, i}^{2}(t+s)=\sum_{k=1}^{K} P^{(k)}\left[r_{s, i}^{(k)}(t+s)-\tilde{r}_{s, i}(t+s)\right]^{2},
\end{aligned}
$$

where $\tilde{\sigma}$ is considered as the degree of risk involved in decision making [1].

Here, if we make a stock portfolio by allocation rates $\left\{d_{i}\right\}$, where $\sum_{i=1}^{N} d_{i}=1$, the expected return rate $\tilde{r}_{s, p}(t+s)$ of the portfolio and the expected risk $\tilde{\sigma}_{s, p}(t+s)$ of it are respectively given by

$$
\begin{aligned}
\tilde{r}_{s, p}(t+s) & =\sum_{i=1}^{N} d_{i} \tilde{r}_{s, i}(t+s), \\
\tilde{\sigma}_{s, p}(t+s) & =\sqrt{\sum_{i=1}^{N} \sum_{j=1}^{N} d_{i} d_{j} \tilde{\sigma}_{s, i j}(t+s)},
\end{aligned}
$$


where $\tilde{\sigma}_{s, i j}(t+s)$ is defined as

$$
\tilde{\sigma}_{s, i j}(t+s)=\sum_{k=1}^{K} P_{i j}^{(k)}\left[r_{s, i}^{(k)}(t+s)-\tilde{r}_{s, i}(t+s)\right] \cdot\left[r_{s, j}^{(k)}(t+s)-\tilde{r}_{s, j}(t+s)\right],
$$

and $P_{i j}^{(k)}$ means the probability that $r_{s, i}(t+s)$ and $r_{s, j}(t+s)$ become $r_{s, i}^{(k)}(t+s)$ and $r_{s, j}^{(k)}(t+s)$.

However, $P^{(k)}, P_{i j}^{(k)}, r_{s, i}^{(k)}(t+s)$, and $r_{s, j}^{(k)}(t+s)$ are unknown. For this reason, the Markowitz portfolio model substitutes historical data of the last $T$ period for the future probability distribution, and then rewrites Eqs. (2), (3), and (6) as follows:

$$
\begin{aligned}
\tilde{r}_{s, i}(t+s) & =\bar{r}_{s, i}(t) \\
& =\frac{1}{T} \sum_{a=0}^{T-1} r_{i}(t-a s) \\
\tilde{\sigma}_{s, i}(t+s) & =\sigma_{s, i}(t) \\
& =\sqrt{\frac{1}{T} \sum_{a=0}^{T-1}\left[r_{s, i}(t-a s)-\bar{r}_{s, i}(t)\right]^{2}} \\
\tilde{\sigma}_{s, i j}(t+s) & =\sigma_{s, i j}(t) \\
& =\frac{1}{T} \sum_{a=0}^{T-1}\left[r_{s, i}(t-a s)-\bar{r}_{s, i}(t)\right] \cdot\left[r_{s, j}(t-a s)-\bar{r}_{s, j}(t)\right] .
\end{aligned}
$$

Then, by substituting these values into Eqs. (4) and (5), we can obtain

$$
\begin{aligned}
& \tilde{r}_{s, p}(t+s)=\sum_{i=1}^{N} d_{i} \bar{r}_{s, i}(t), \\
& \tilde{\sigma}_{s, p}^{2}(t+s)=\sum_{i=1}^{N} \sum_{j=1}^{N} d_{i} d_{j} \sigma_{s, i j}(t) .
\end{aligned}
$$

Moreover, if we allocate $\left\{d_{i}\right\}$ so as to maximize $\tilde{r}_{s, p}(t+s)$ and minimize $\tilde{\sigma}_{s, p}(t+s)$, this investment can be reasonable. Therefore, we apply a typical measure: the Sharpe ratio $S_{r}[6]$ given by

$$
S_{r}=\frac{\tilde{r}_{p}-r_{f}}{\tilde{\sigma}_{p}},
$$

and optimize $\left\{d_{i}^{*}\right\}$ so as to maximize $S_{r}$. Here, $r_{f}$ is a risk-free rate, and the overnight unsecured call rate [7] is used for it. If $\tilde{r}_{p}<0$, we build a selling portfolio to realize a positive $\tilde{r}_{p}$. In this section, because we do not consider the trading-unit system and can trade the odd amount of each stock, the feasible solutions of $\left\{d_{i}\right\}$ are continuous and maximizing $S_{r}$ is a convex quadratic programming problem, which can be solved by the interior point method.

\section{Our nonlinear portfolio model}

In this section, we modify the estimations in Eqs. (7)-(9) of the Markowitz portfolio model by using time-series prediction models.

\subsection{Time-series prediction models}

In the Markowitz mean-variance portfolio model, we can see that $\tilde{r}_{s, i}(t+s)$ of Eq. (7) is predicted by simple moving average (SMA) prediction. However, if a future movement depends on the past movements more complexly and this relationship can be approximated by a super plane, we can expect the future return rate of Eq. (7) by the autoregressive (AR) model:

$$
\tilde{r}_{s, i}(t+s)=\sum_{a=0}^{T-1} \beta_{a} r_{s, i}(t-a s)+\beta_{T} .
$$


Here, to estimate model parameters $\beta_{a}(a=0,1, \cdots, T)$, we apply the least square method to the learning data, which are historical data of the recent $L$ period. Then, Eq. (13) can be rewritten in a vector form:

$$
\boldsymbol{Y}=\boldsymbol{X} \boldsymbol{F},
$$

where

$$
\begin{aligned}
\boldsymbol{Y} & =\left[r_{s, i}(t), r_{s, i}(t-s), r_{s, i}(t-2 s), \cdots, r_{s, i}(t-(L-1)+T s)\right]^{\mathrm{t}}, \\
\boldsymbol{X} & =\left[\begin{array}{cc}
\boldsymbol{r}_{s, i}(t-s) & 1 \\
\boldsymbol{r}_{s, i}(t-2 s) & 1 \\
\vdots & \vdots \\
\boldsymbol{r}_{s, i}(t-(L-1)+(T-1) s) & 1
\end{array}\right], \\
\boldsymbol{r}_{s, i}(t-a s) & =\left[r_{s, i}(t-a s), r_{s, i}(t-(a+1) s), r_{s, i}(t-(a+2) s), \cdots, r_{s, i}(t-(a+(T-1)) s)\right], \\
\boldsymbol{F} & =\left[\beta_{0}, \beta_{1}, \cdots, \beta_{T}\right]^{t} .
\end{aligned}
$$

Therefore, the model parameters of Eq. (13) can be estimated by

$$
\tilde{\boldsymbol{F}}=\left[\boldsymbol{X}^{\mathrm{t}} \boldsymbol{X}\right]^{-1} \boldsymbol{X}^{\mathrm{t}} \boldsymbol{Y} .
$$

The AR model is classified into a linear prediction model because it approximates all learning data by a regression plane.

In addition, we can also use a nonlinear prediction model which can realize not only a regression plane but also a regression surface. First, we rewrite Eq. (13) as

$$
\tilde{r}_{s, i}(t+s)=\left[\boldsymbol{r}_{s, i}(t) 1\right] \cdot \boldsymbol{F} .
$$

Here, we want to change the model parameters $\boldsymbol{F}$ according to similar input variables $\boldsymbol{r}_{s, i}(t)$ because similar outputs are generated by similar inputs, especially if this system has a nonlinear dynamics $\boldsymbol{F}$. Namely, we consider similar historical data $\boldsymbol{r}_{s, i}(t)$ more important to estimate $\boldsymbol{F}$, and we apply the weighted least squares method according to the distance $l\left(t_{a s}\right)$ :

$$
l\left(t_{a s}\right)=\left|\boldsymbol{r}_{s, i}(t)-\boldsymbol{r}_{s, i}(t-a s)\right|,
$$

where $\boldsymbol{r}_{s, i}(t)$ is a target input and $\boldsymbol{r}_{s, i}(t-a s)$ are the other learning data. Then, the weighted factor $w\left(t_{a s}\right)$ is given by

$$
w\left(t_{a s}\right)=\exp \left(-l\left(t_{a s}\right)\right) .
$$

Next, we prepare the following diagonal matrix:

$$
\boldsymbol{W}=\left[\begin{array}{cccc}
w\left(t_{s}\right) & 0 & \cdots & 0 \\
0 & w_{i}\left(t_{2 s}\right) & \cdots & 0 \\
\vdots & \vdots & \ddots & \vdots \\
0 & \cdots & 0 & w_{i}\left(t_{(L-1)+(T-1) s}\right)
\end{array}\right]
$$

and then, the model parameters of Eq. (13) can be estimated by

$$
\tilde{\boldsymbol{F}}=\left[\boldsymbol{X}^{\mathrm{t}} \boldsymbol{W}^{\mathrm{t}} \boldsymbol{W} \boldsymbol{X}\right]^{-1} \boldsymbol{X}^{\mathrm{t}} \boldsymbol{W}^{\mathrm{t}} \boldsymbol{W} \boldsymbol{Y} .
$$

Here, this estimation of $\tilde{\boldsymbol{F}}$ is approximated locally with a super plane, but the slope of each plane changes locally according to the state of $\boldsymbol{r}_{s, i}(t)$. Namely, this approximation corresponds to a nonlinear regression with a super surface globally. In the present study, we call it the nonlinear autoregressive (NAR) model, which is extended from the AR model by the local linear approximation method [8].

Then, we evaluate the prediction accuracy $\xi$ by using the correlation coefficient: 


$$
\xi=\frac{\sum_{t}(r(t)-\langle r\rangle)(\tilde{r}(t)-\langle\tilde{r}\rangle)}{\sqrt{\sum_{t}(r(t)-\langle r\rangle)^{2}} \sqrt{\sum_{t}(\tilde{r}(t)-\langle\tilde{r}\rangle)^{2}}},
$$

where $\tilde{r}(t)$ is the predicted value, $r(t)$ is its true value, and $\langle\cdot\rangle$ means the average value.

\subsection{Modification of the Markowitz portfolio model}

In order to apply not only the SMA prediction used for the original Markowitz portfolio model but also any other prediction models, we have to modify Eqs. (7)-(9) by reconsidering them from their original definitions of Eqs. (2), (3) and (6).

According to Eq. (2), because the expected return rate $\tilde{r}_{s, i}(t+s)$ means the most probable future, we can replace it by the predicted value. Here, this idea is not too strange because Eq. (7) of the original model can be considered as a predicted value by the SMA prediction. Then, by substituting the predicted value into Eq. (4), we can calculate the expected return rate of a portfolio $\tilde{r}_{s, p}(t+s)$.

Moreover, in Eq. (3), we can say that the Markowitz portfolio model regards the risk $\tilde{\sigma}_{s, i}(t+s)$ as the expected value of prediction errors. Therefore, we denote the prediction error $r_{s, i}^{(k)}(t+s)-\tilde{r}_{s, i}(t+s)$ as $\sigma_{s, i}^{(k)}(t+s)$, and then Eq. (3) is rewritten by

$$
\tilde{\sigma}_{s, i}(t+s)=\sqrt{\sum_{k=1}^{K} P^{(k)}\left[\sigma_{s, i}^{(k)}(t+s)\right]^{2}} .
$$

Although $P^{(k)}$ and $\sigma_{s, i}^{(k)}(t+s)$ are unknown similarly to the Markowitz portfolio model, these are estimated by historical prediction errors $r_{s, i}\left(t^{\prime}\right)-\tilde{r}_{s, i}\left(t^{\prime}\right)\left(t^{\prime} \leq t\right)$. Therefore, Eq. (3) can be rewritten by

$$
\tilde{\sigma}_{s, i}(t+1)=\sqrt{\frac{1}{T} \sum_{a=0}^{T-1}\left[r_{s, i}(t-a s)-\tilde{r}_{s, i}(t-a s)\right]^{2}} .
$$

On the other hand, the Markowitz portfolio model focuses on only $r_{s, i}^{(k)}(t+s)$ included in $\sigma_{s, i}^{(k)}(t+s)$ when Eq. (3) is modified into Eq. (8). However, in the case of predicting future returns aggressively like our study, because the essence of the risk is based on prediction errors, we estimate the whole of $\sigma_{s, i}^{(k)}(t+s)$ by past prediction errors.

Similarly, the covariance of Eq. (6) is rewritten by

$$
\begin{aligned}
\tilde{\sigma}_{s, i j}(t+s) & =\sum_{k=1}^{K} P_{i j}^{(k)} \sigma_{s, i}^{(k)}(t+s) \sigma_{s, j}^{(k)}(t+s) \\
& =\frac{1}{T} \sum_{a=0}^{T-1}\left[r_{s, i}(t-a s)-\tilde{r}_{s, i}(t-a s)\right]\left[r_{s, j}(t-a s)-\tilde{r}_{s, j}(t-a s)\right]
\end{aligned}
$$

Then, we can estimate $\tilde{\sigma}_{s, p}(t+s)$ by substituting this $\tilde{\sigma}_{s, i j}(t+s)$ into Eq. (5).

However, in actual use, we have to take care of the data length $T$ in Eq. (13). As $T$ is smaller, it causes that at least one of the covariance of Eq. (28) becomes a large negative value accidentally. For example, if the two indexes $i=1$ and $j=2$ have completely negative correlation, their covariance becomes $\tilde{\sigma}_{12}=-\tilde{\sigma}_{1} \tilde{\sigma}_{2}$. In this case, by setting allocation rates except $d_{1}$ and $d_{2}$ to 0 , Eq. (11) is rewritten as follows:

$$
\begin{aligned}
\tilde{\sigma}_{p}^{2} & =\sum_{i=1}^{N} \sum_{j=1}^{N} d_{i} d_{j} \tilde{\sigma}_{i j}=\sum_{i=1}^{N} d_{i}^{2} \tilde{\sigma}_{i}^{2}+2 \sum_{i=1}^{N} \sum_{j>i}^{N} d_{i} d_{j} \tilde{\sigma}_{i j} . \\
& =d_{1}^{2} \tilde{\sigma}_{1}^{2}+d_{2}^{2} \tilde{\sigma}_{2}^{2}+2 d_{1} d_{2} \tilde{\sigma}_{12}=d_{1}^{2} \tilde{\sigma}_{1}^{2}+d_{2}^{2} \tilde{\sigma}_{2}^{2}-2 d_{1} d_{2} \tilde{\sigma}_{1} \tilde{\sigma}_{2} \\
& =\left(d_{1} \tilde{\sigma}_{1}-d_{2} \tilde{\sigma}_{2}\right)^{2}
\end{aligned}
$$



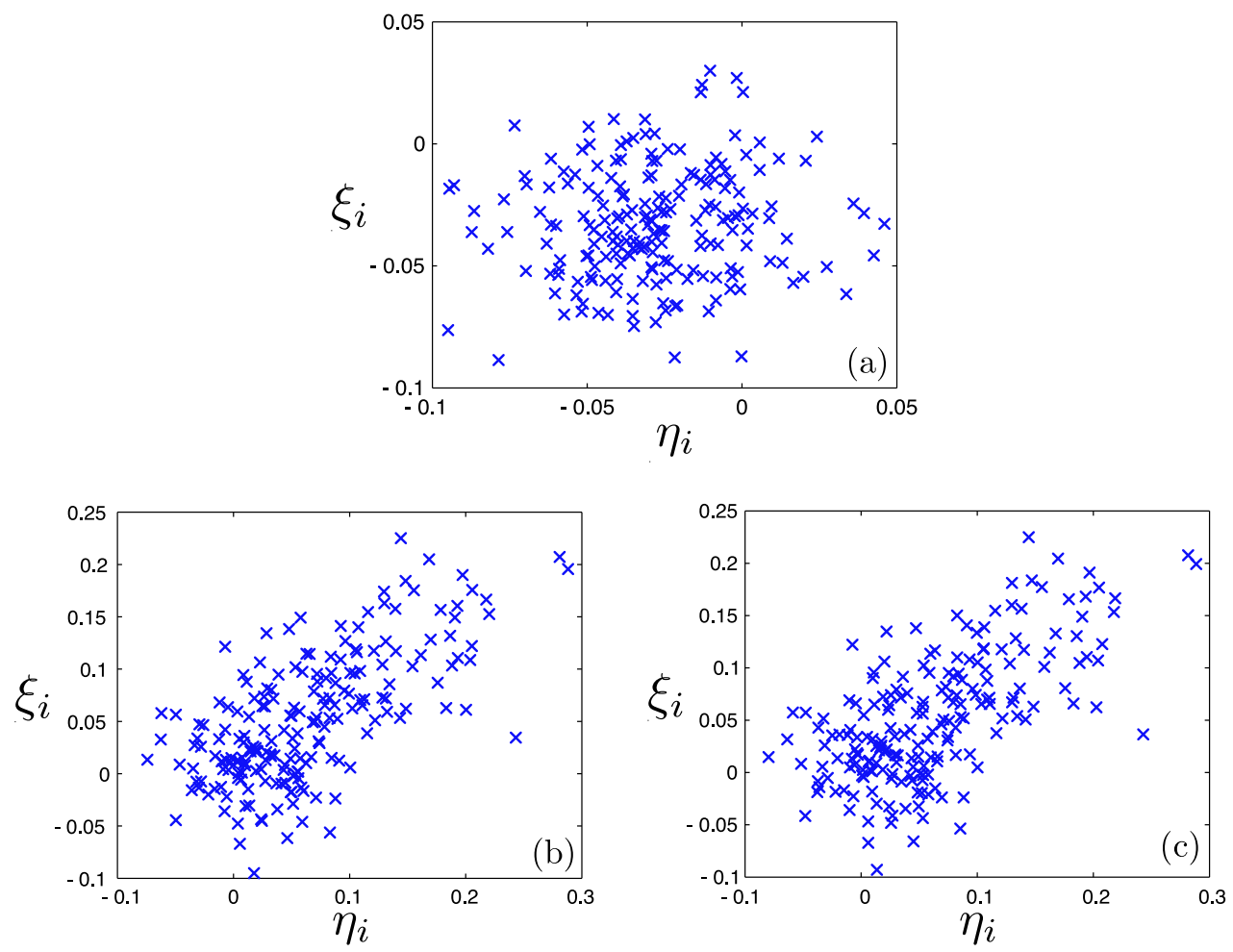

Fig. 1. Correlation diagrams between the likelihood $\left\{\eta_{i}\right\}$ and the prediction accuracy $\left\{\xi_{i}\right\}$ given by (a) the SMA prediction, (b) the AR prediction, and (c) the NAR prediction. Each correlation is (a) 0.120, (b) 0.661, and (c) 0.660 .

and therefore,

$$
\tilde{\sigma}_{p}=\left|d_{1} \tilde{\sigma}_{1}-d_{2} \tilde{\sigma}_{2}\right| .
$$

Here, if we set like $d_{1}: d_{2}=\tilde{\sigma}_{2}: \tilde{\sigma}_{1}$ and $d_{i \notin\{1,2\}}=0$, we can realize a no-risk portfolio of $\tilde{\sigma}_{p}=0$, and the Sharpe ratio of Eq. (12) diverges to infinity. Namely, if the prediction errors of any two stocks have a negative correlation accidentally, the best allocation rates to maximize $S_{r}$ can be decided only by $\tilde{\sigma}_{1}$ and $\tilde{\sigma}_{2}$. However, this portfolio is definitely false because any of the expected return rates $\left\{\tilde{r}_{i}\right\}$ are not applied. The danger of this false portfolio becomes higher as the number of $T$ of Eq. (28) becomes smaller. Therefore, we use enough large number of $T$ so as to make $\max \left\{S_{r}\right\}$ converge to a finite value.

\subsection{Investment simulations}

To confirm the validity of the modification of Secs. 3.1 and 3.2, we performed investment simulations with 200 kinds of daily stock data [9]. The Investment period is from April 8, 2000 until past five years, that is, for 1250 business days except for Saturdays, Sundays, and holidays. Then, the learning data is selected for five years before April 7, 2000 (i.e., $L=1250$ ). In this section, we do not yet consider stock-trading commissions and the minimum trading amount of stocks. Then, we set $s=1$ to predict tomorrow's return rates $\left\{r_{1, i}(t+1)\right\}$ of 200 stocks, and we compose a daily portfolio and rebalance it everyday.

Figure 1 shows correlation diagrams for the likelihoods $\left\{\eta_{i}\right\}$ and the prediction accuracies $\left\{\xi_{i}\right\}$ of each prediction model. Here, the likelihoods $\left\{\eta_{i}\right\}$ means the prediction accuracy of the learning data. Namely, because we have their true values, we can know the validity of prediction models as the prediction accuracy before investments. On the other hand, the prediction accuracy $\left\{\xi_{i}\right\}$ is given by the test data of the investment period after the learning data, and $\left\{\xi_{i}\right\}$ is originally unknown before investments. Therefore, if there exists a positive correlation between the unknown $\left\{\xi_{i}\right\}$ and the known $\left\{\eta_{i}\right\}$, we can expect the unknown information beforehand.

Actually, in Figs. 1(b) and (c), we can confirm that the prediction accuracy $\left\{\xi_{i}\right\}$ has enough correlation with the likelihood $\left\{\eta_{i}\right\}$ except for the SMA prediction of the Markowitz portfolio model 

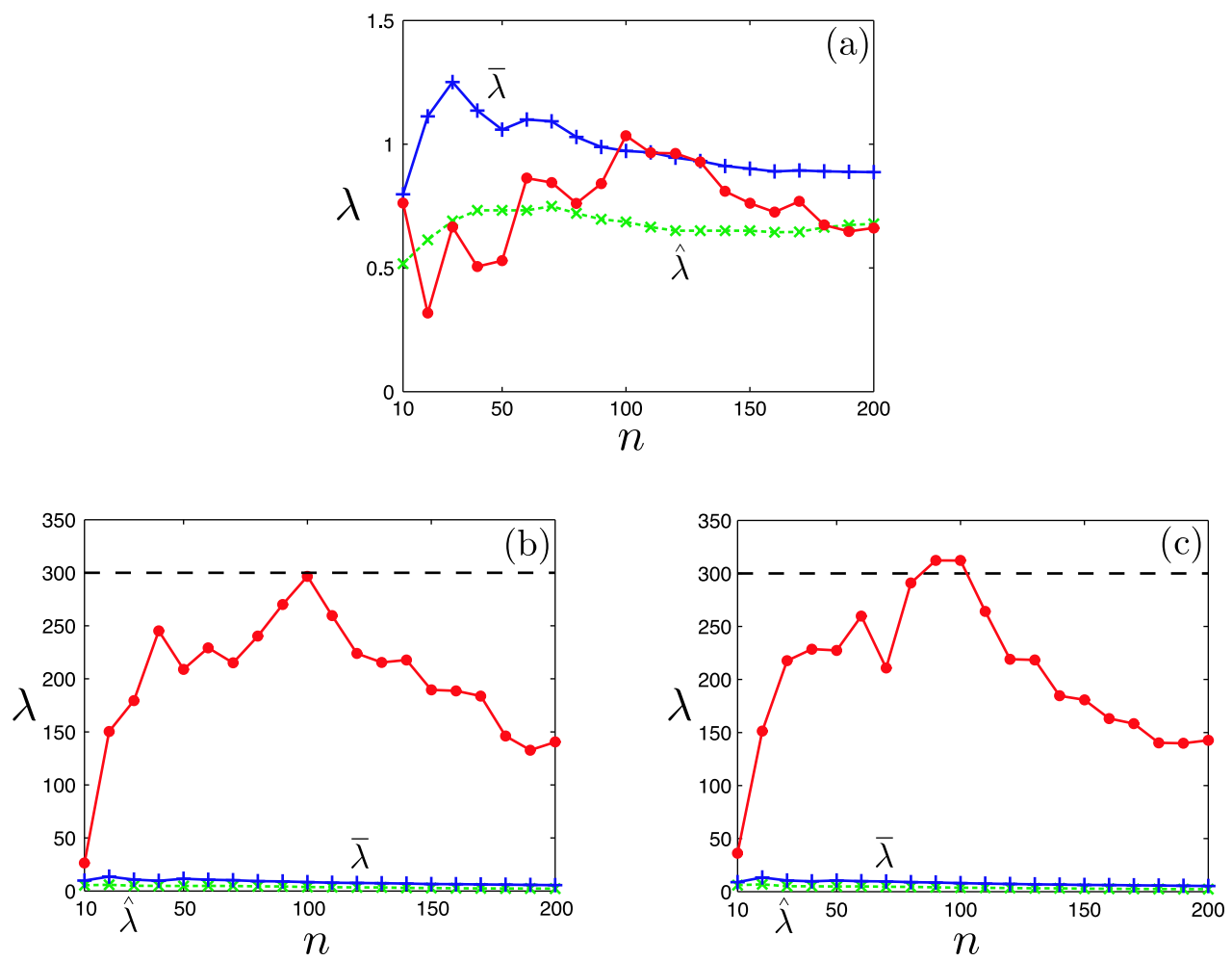

Fig. 2. Results of the asset growth rate $\lambda$ (solid-dot lines) of the multi-stock portfolio where $n$ stocks were selected by the likelihood $\left\{\eta_{i}\right\}$ based on (a) the SMA prediction of the Markowitz portfolio model, (b) the AR prediction of our portfolio model, and (c) the NAR prediction of our portfolio model. The index $\bar{\lambda}$ means the mean value of $\left\{\lambda_{i}\right\}$ given by each single stock investment without any portfolios, and $\hat{\lambda}$ means the median value of them.

shown in Fig. 1(a). Therefore, to make a multi-stock portfolio with $n$ stocks, we preferentially selected more predictable stocks having higher likelihood of each prediction model for the learning data.

Figure 2 shows investment performance of the multi-stock portfolio where the selected $n$ stocks depend on each prediction model. Then, the allocation rates $\left\{d_{i}\right\}$ of the selected $n$ stocks were rebalanced everyday by the Sharpe ratio $S_{r}$ of Eq. (12), but stock-trading commissions were ignored in this section. As an index to evaluate investment performance, we calculated the asset growth rate $\lambda$ :

$$
\lambda=\frac{M(*)}{M(0)}
$$

where $M(0)$ means the amount of the initial asset and $M(*)$ means that of the final asset after the investment simulation.

In addition, to confirm the portfolio effect, we also evaluated the benchmark performance by trading a single stock without making any portfolios, that is, we invested each stock independently according to the predicted future return $\tilde{r}_{1, i}(t+1)$. In this single stock investment, if $\tilde{r}_{1, i}(t+1)>0$, we buy the $i$ th stock; if $\tilde{r}_{1, i}(t+1)<0$, we sell the $i$ th stock. And then, at the time of $t+1$, we close the position and make a new position based on $\tilde{r}_{1, i}(t+2)$.

As shown in Fig. 2, each $\lambda$ looks convex and has a peak. If the number of stocks $n$ to compose a portfolio increases, we can reduce the total risk by the portfolio effect. However, as $n$ is larger, we use even undesirable stocks whose prediction accuracies are low. Therefore, the performance of this portfolio decreases. Next, we can see that $\lambda$ of Fig. 2(a) is the smallest of the three Figures. This means that the prediction accuracy by the SMA prediction is too bad to make a portfolio and rebalance it. Moreover, $\bar{\lambda}$ of the benchmark is higher than $\lambda$ of the portfolio because the single stock investment can select a sell or a buy position to each stock, and therefore it has larger flexibility of dealing patterns. On the other hand, as shown in Figs. 2(b) and (c), we can confirm strong improvement of $\lambda$ by 
applying the AR or the NAR prediction model to modify the Markowitz portfolio model. This means that our portfolio model can realize better predictions by using advanced prediction models and can reduce the total risk of the portfolio effectively by selecting predictable stocks having higher likelihood of each prediction model. Especially, the NAR (nonlinear) prediction shows a slight advantage to the AR (linear) prediction, and therefore we focus on the only NAR prediction for our portfolio model in the following sections.

\section{Portfolio rebalancing strategy in real investment situations}

In the previous sections, we had considered an ideal situation where stock-trading commotions were free and the minimum trading amount of each stock was ignored. However, we cannot avoid these obstructions to discuss a useful portfolio model for real investment situations. For this reason, we introduce long-term prediction method in Sec.4.1 to reduce the frequency of portfolio rebalancing and rebalancing costs. Moreover, we modify the Sharpe ratio in Sec.4.2 to perform the portfolio optimization based on rebalancing costs.

\subsection{Long-term prediction to reduce the rebalancing frequency}

By increasing $s$ of Eqs. (7) and (13), we can perform long-term prediction and estimate the $s$-steps future return rate $\tilde{r}_{s, i}(t+s)$, which can reduce the frequency of portfolio rebalancing to save its rebalancing costs.

However, if we apply a large $s$ to prediction models, the amount of the learning data is reduced, and consequently it becomes hard to estimate the model parameters $\beta_{a}(a=0,1, \cdots, T)$. For this reason, we introduce a more advanced algorithm for a long-term prediction, modifying Eqs. (13), (15), and (16) into

$$
\begin{aligned}
\tilde{r}_{s, i}(t+s) & =\sum_{a=0}^{T-1} \beta_{a} r_{1, i}(t-a)+\beta_{T}, \\
\boldsymbol{Y} & =\left[r_{s, i}(t), r_{s, i}(t-1), \cdots, r_{s, i}(t-(L-T)+s)\right]^{\mathrm{t}}, \\
\boldsymbol{X} & =\left[\begin{array}{cc}
\boldsymbol{r}_{1, i}(t-s) & 1 \\
\boldsymbol{r}_{1, i}(t-(s+1)) & 1 \\
\vdots & \vdots \\
\boldsymbol{r}_{1, i}(t-(L-T)) & 1
\end{array}\right] .
\end{aligned}
$$

Then, by substituting $\boldsymbol{Y}$ and $\boldsymbol{X}$ into Eq. (24), we can get the model parameters $\boldsymbol{F}=\left\{\beta_{0}, \beta_{1}, \cdots, \beta_{T}\right\}$ for Eq. (13). In Eqs. (34) and (16), each $\boldsymbol{X}$ has the following learning data set: $\left\{\boldsymbol{r}_{1, i}(t-s), \boldsymbol{r}_{1, i}(t-\right.$ $\left.(s+1)), \boldsymbol{r}_{1, i}(t-(s+2)), \cdots\right\}$ and $\left\{\boldsymbol{r}_{s, i}(t-s), \boldsymbol{r}_{s, i}(t-2 s), \boldsymbol{r}_{p, i}(t-3 s), \cdots\right\}$, respectively. Therefore, as $s$ is larger, the learning data of Eq. (15) are more omitted. In the present study, we call the prediction method based on Eq. (34) as the advanced NAR prediction, which can avoid the reduction of the learning data.

Next, to confirm the efficacy of the advanced NAR prediction, we performed long-term predictions to the same stock data $(i=1 \sim 200)$ as Sec.3.3., and calculated the set of the prediction accuracies $\left\{\xi_{i}\right\}$ and that of the likelihoods $\left\{\eta_{i}\right\}$. In Fig. 3(a), we can confirm that the advanced NAR prediction improved the NAR prediction and realized the best prediction accuracy. Then, as $s$ is larger, each prediction accuracy is more reduced, which means that longer-term prediction is more difficult.

Next, Fig. 3(b) shows the correlation coefficient $C$ between the likelihoods $\left\{\eta_{i}\right\}$ and the prediction accuracies $\left\{\xi_{i}\right\}$. As mentioned before, if the correlation coefficient $C$ is high, we can select predictable stocks in advance. As the same as Fig. 3(a), shorter-term predictions show better results, that is, higher correlation $C$. Then, because long-term prediction is difficult, the correlation $C$ is reduced by a large $s$.

Because we can confirm the positive correlation in Fig. 3(b), we selected the most predictable $n$ stocks based on the largest likelihoods $\left\{\eta_{i}\right\}$ and evaluated the prediction accuracies $\left\{\xi_{i}\right\}$ of the selected $n$ stocks. This result is shown in Fig. 4. In each figure, we can see larger prediction accuracy as $s$ 

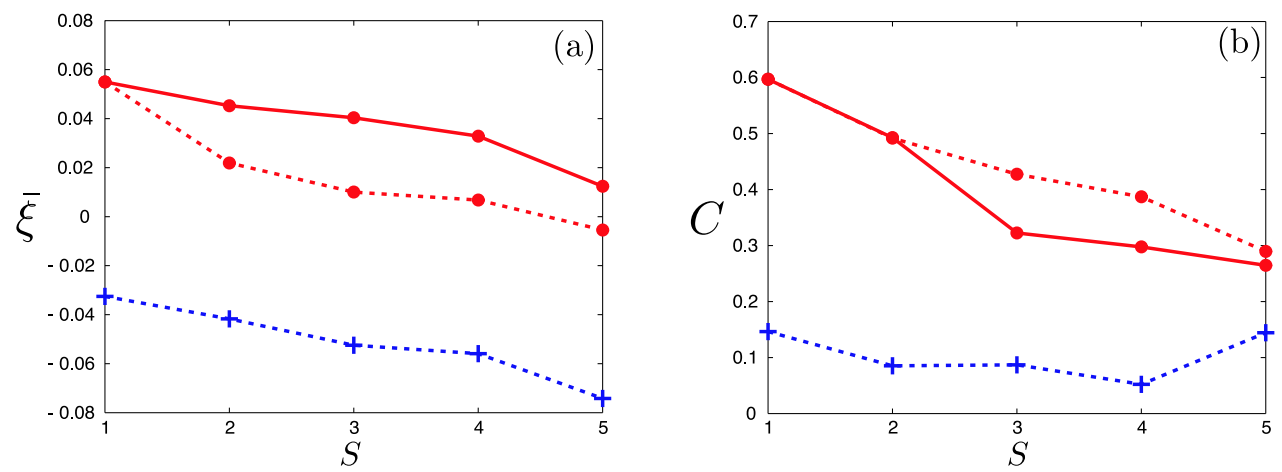

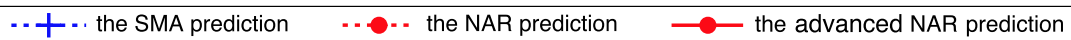

Fig. 3. Results of long-term ( $s$ steps) predictions: (a) the mean value $\bar{\xi}$ of the prediction accuracies $\left\{\xi_{i}\right\}$ and (b) the correlation coefficient $C$ between the likelihoods $\left\{\eta_{i}\right\}$ and the prediction accuracies $\left\{\xi_{i}\right\}$.
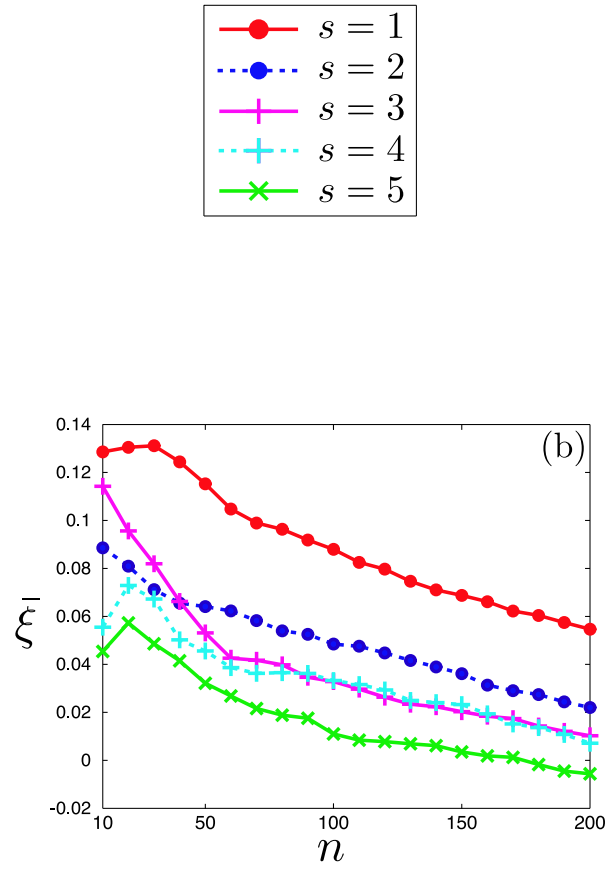
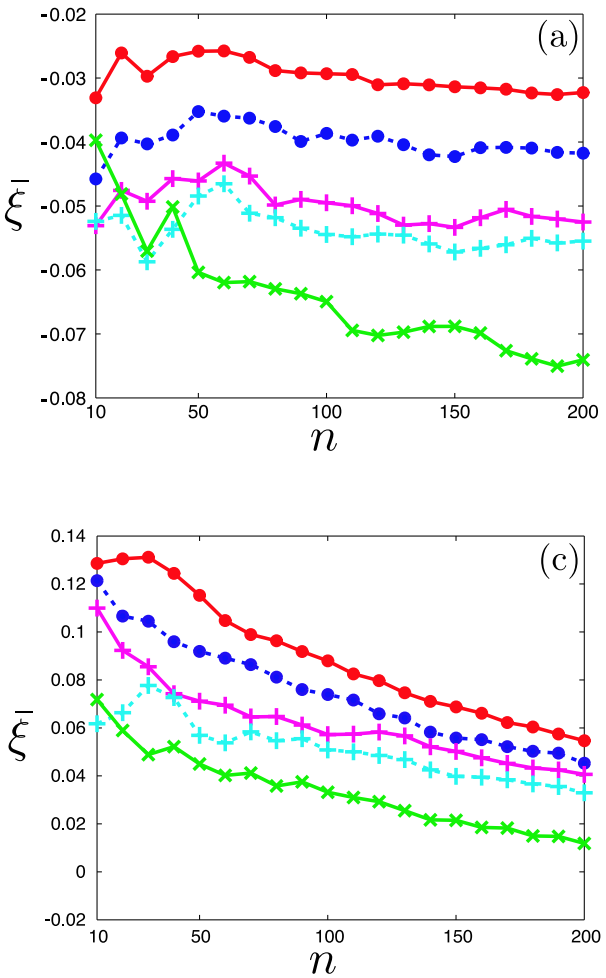

Fig. 4. Results of the best $n$ stocks based on the likelihoods $\left\{\eta_{i}\right\}$ given by each prediction model: (a) the SMA prediction, (b) the NAR prediction, and (c) the advanced NAR prediction.

is smaller. This means that we can select predictable stocks to some degree especially by nonlinear prediction methods. Here, it is natural that each prediction accuracy goes down with larger $n$ because unpredictable stocks are more included. Moreover, this selection works best with the advantage NAR prediction even if $s$ is large as shown in Fig. 4(c). Therefore, in the following sections, we apply the advanced NAR prediction to our nonlinear portfolio model instead of the NAR prediction.

\subsection{Modification of the Sharpe ratio by rebalancing costs}

Next, we introduce stock-trading commissions for portfolio rebalancing. As mentioned before, the Sharpe ratio [6] is generally used for an evaluation function to optimize a portfolio and rebalance it. However, because the original Sharpe ratio does not consider any stock-trading commissions, we modify it as follows: 

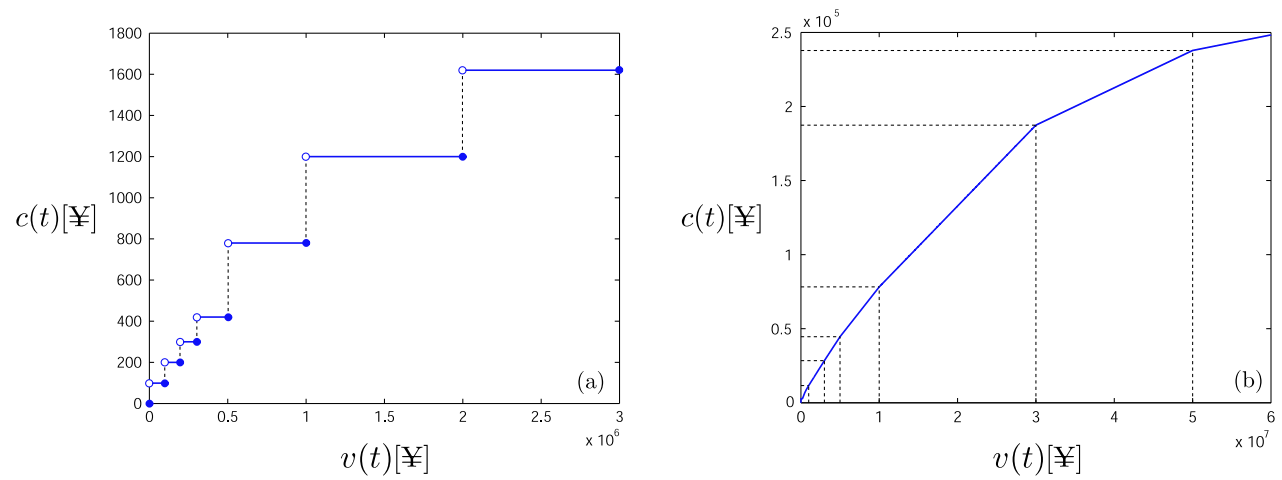

Fig. 5. Examples of cost functions $f$ for the volume of portfolio rebalancing $v(t)$ : (a) Okasan Online Securities (online) and (b) Nomura Securities (over the counter). In Fig. (a), if $v(t)>3 \times 10^{6}[¥]$, the commission $c(t)$ is increased by $420[¥]$ for every additional $1 \times 10^{6}[¥]$.

$$
S_{r}^{\prime}=\frac{M(t)-\tilde{r}_{s, p}(t+s)-M(t) r_{f}(t+s)-c(t)}{M(t) \tilde{\sigma}_{s, p}(t+s)},
$$

where $M(t)$ means the amount of assets at the time of $t$, and $c(t)$ means the total cost for a portfolio rebalancing.

Next, to calculate this total cost, we note that the allocation rates optimized before are automatically changed everyday because every stock price changes. That is, $\left\{d_{i}(t)\right\} \neq\left\{d_{i}^{*}(t-s)\right\}, s \geq 1$, where $\left\{d_{i}(t)\right\}$ means the present allocation rates and $\left\{d_{i}^{*}(t-s)\right\}$ means the past allocation rates optimized before. Therefore, the total volume of selling and buying stocks for a portfolio rebalancing $v(t)$ and its total commission $c(t)$ are given by

$$
\begin{aligned}
v(t) & =M(t) \sum_{i=1}^{N}\left|d_{i}^{*}(t)-d_{i}(t)\right| \\
c(t) & =f(v(t))
\end{aligned}
$$

where $\left\{d_{i}^{*}(t)\right\}$ means new allocation rates optimized by maximizing Eq. (35) for portfolio rebalancing, and $f$ means a commission function. In this study, we use the commission function of a online trading company [10] shown in Fig. 5(a) because the commission is generally cheaper than over-the-counter companies like Fig. 5(b).

If we apply a nonlinear cost function like Fig. 5 to the modified Sharpe ratio $S_{r}^{\prime}$ of Eq. (35), maximizing $S_{r}^{\prime}$ is not a convex quadratic programming problem, and therefore we cannot apply the interior point method. For this reason, we collected some quasi-optimum solutions by the multi-start local search method, and then we decided the best solution $\left\{d_{i}^{*}(t)\right\}$ of these solutions.

Moreover, in this study, we also consider the trading-unit system for real investment situations, where we have to trade 1000 stocks as one unit at Tokyo Stock Exchange. Therefore, $\left\{d_{i}^{*}(t)\right\}$ should be optimized discretely because the solution space of $\left\{d_{i}(t)\right\}$ is discrete. For this reason, the best solution $\left\{d_{i}^{*}\right\}$ optimized above was rounded up or rounded down to obtain feasible solutions based on the trading-unit system. Because this optimization is the 0-1 integer programming problem, we applied the genetic algorithm to maximize $S_{r}^{\prime}$ of Eq. (35).

\subsection{Investment simulations}

We perform investment simulations to confirm the validity of our nonlinear portfolio model and our modified Sharpe ratio. First, to focus on our portfolio model, we applied the original $S_{r}$, which cannot consider any stock-trading commissions, but these commissions were applied in every investment simulation. Figure 6 shows the asset growth rate $\lambda$ given by the Markowitz portfolio model and our nonlinear portfolio model. Moreover, to discuss the best frequency of portfolio rebalancing and the influence of the trading-unit system, we changed the prediction step $s$ and the amount of initial assets $M(0)$. Here, $s$ corresponds to the frequency of portfolio rebalancing. If $s=5$, we make a portfolio for the next five days and then rebalance it every five days during the investment period. 

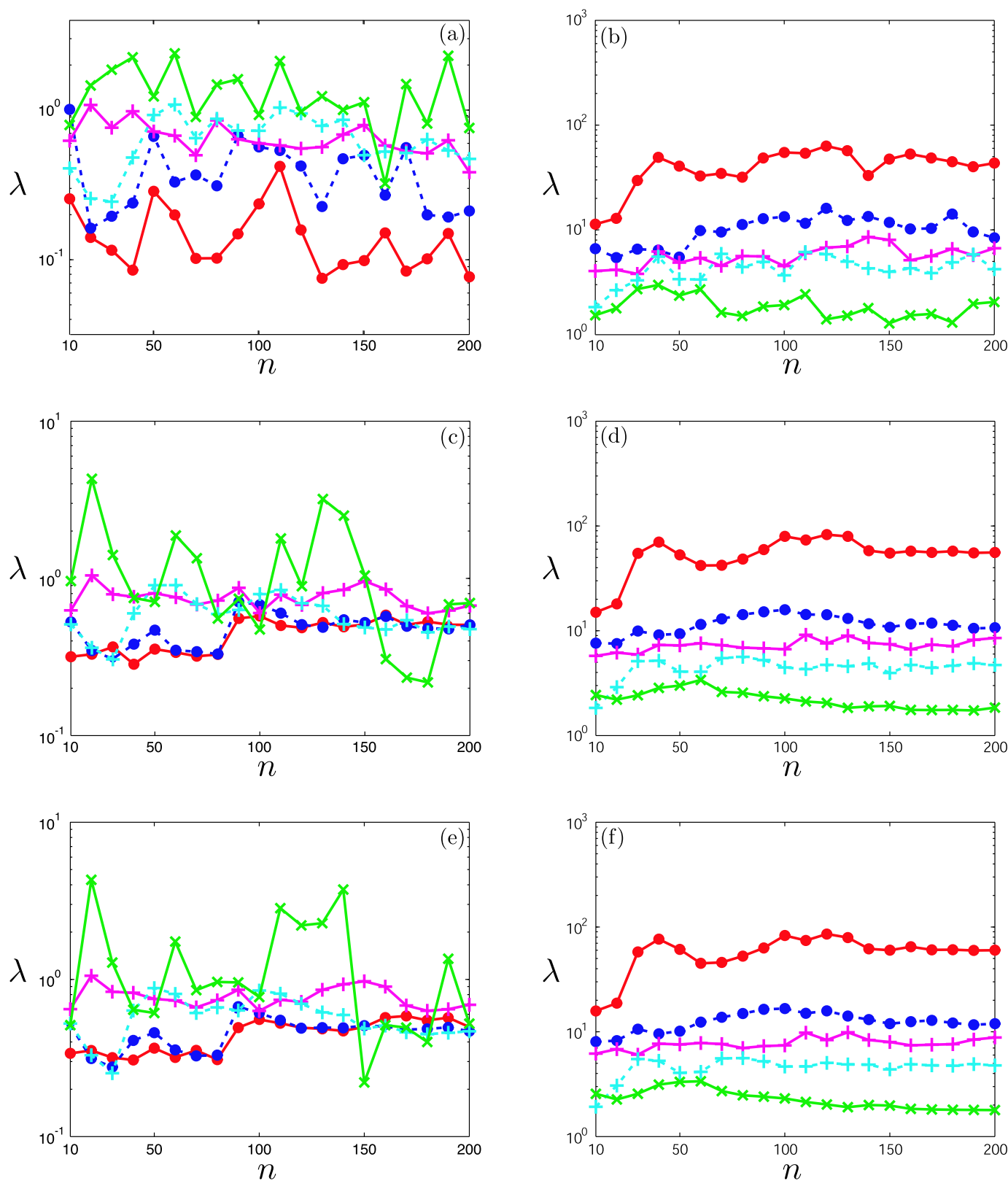

$$
\begin{array}{ll}
\longrightarrow & -\multimap s=1 \text { (Everyday) } \\
-+-s=4 \text { (Every four days) } & \leftarrow s=5 \text { (Every five days) }
\end{array}
$$

Fig. 6. Results of the asset growth rate $\lambda$ in real investment situations, where stock-trading commissions $c(t)$ and the trading-unit system are inevitable. The Markowitz portfolio model was applied in (a), (c), and (e). Then, our nonlinear portfolio model was applied in (b), (d), and (f). In each model, the original Sharp ratio $S_{r}$ was used. Then, the initial asset $M(0)$ was set to $10^{6}[¥]$ in (a) and (b), $10^{7}[¥]$ in $(\mathrm{c})$ and $(\mathrm{d})$, and $10^{8}[¥]$ in (e) and (f). The prediction step $s$ corresponds to the interval of each portfolio rebalancing.

In Figs. 6(a), (c), and (e) given by the Markowitz portfolio model, $\lambda$ is more reduced as $s$ is smaller because shorter-term rebalancing requires us to pay more stock-trading commotions. Moreover, as shown in Fig. 4(a), the prediction accuracy $\bar{\xi}$ of the SMA prediction was almost zero. Namely, the Markowitz portfolio model did not work well, and its portfolio rebalancing was useless to follow market trends. After all, stock-trading commissions reduces the investment performance $\lambda$. Therefore, longerterm rebalancing by using a larger $s$ shows better $\lambda$ because it can reduce rebalancing costs. These results support the previous studies that explain the uselessness of the active investment strategy because stock-price movements are considered as the random walk [11]. 
$\longrightarrow \quad S_{r}^{\prime} \rightarrow$ Max and $M(0)=10^{8}[¥]$

十 $S_{r}^{\prime} \rightarrow$ Max and $M(0)=10^{7}[¥]$

$\longrightarrow \quad S_{r}^{\prime} \rightarrow$ Max and $M(0)=10^{6}[¥]$

$-\rightarrow S_{r} \rightarrow \operatorname{Max}$ and $M(0)=10^{8}[¥]$

- + - $S_{r} \rightarrow$ Max and $M(0)=10^{7}[¥]$

$--\star-\quad S_{r} \rightarrow \operatorname{Max}$ and $M(0)=10^{6}[¥]$
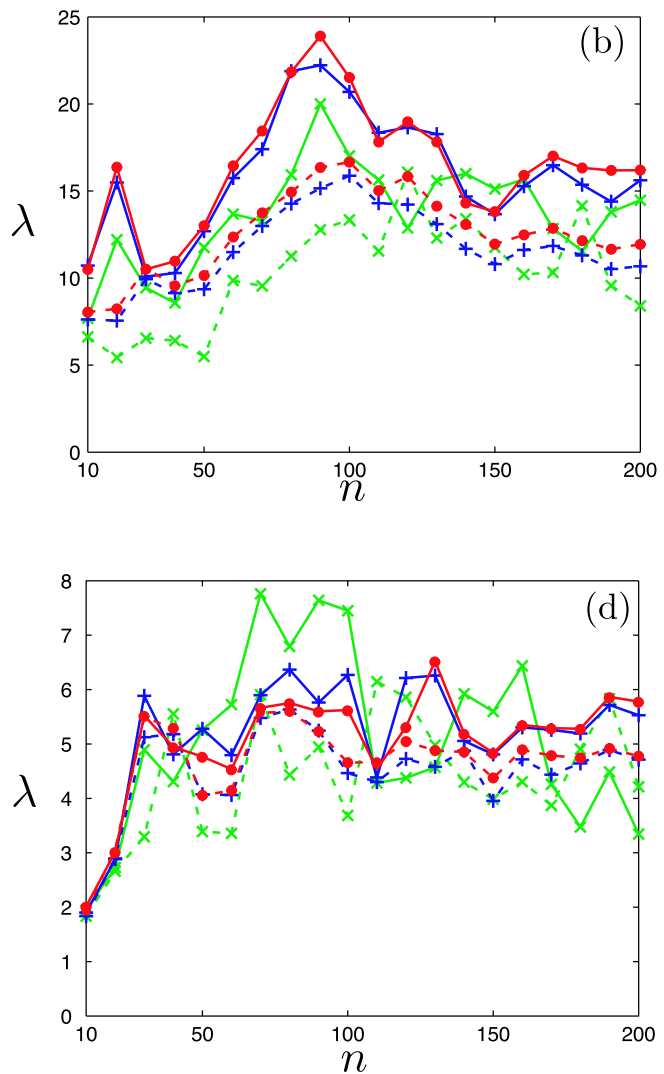
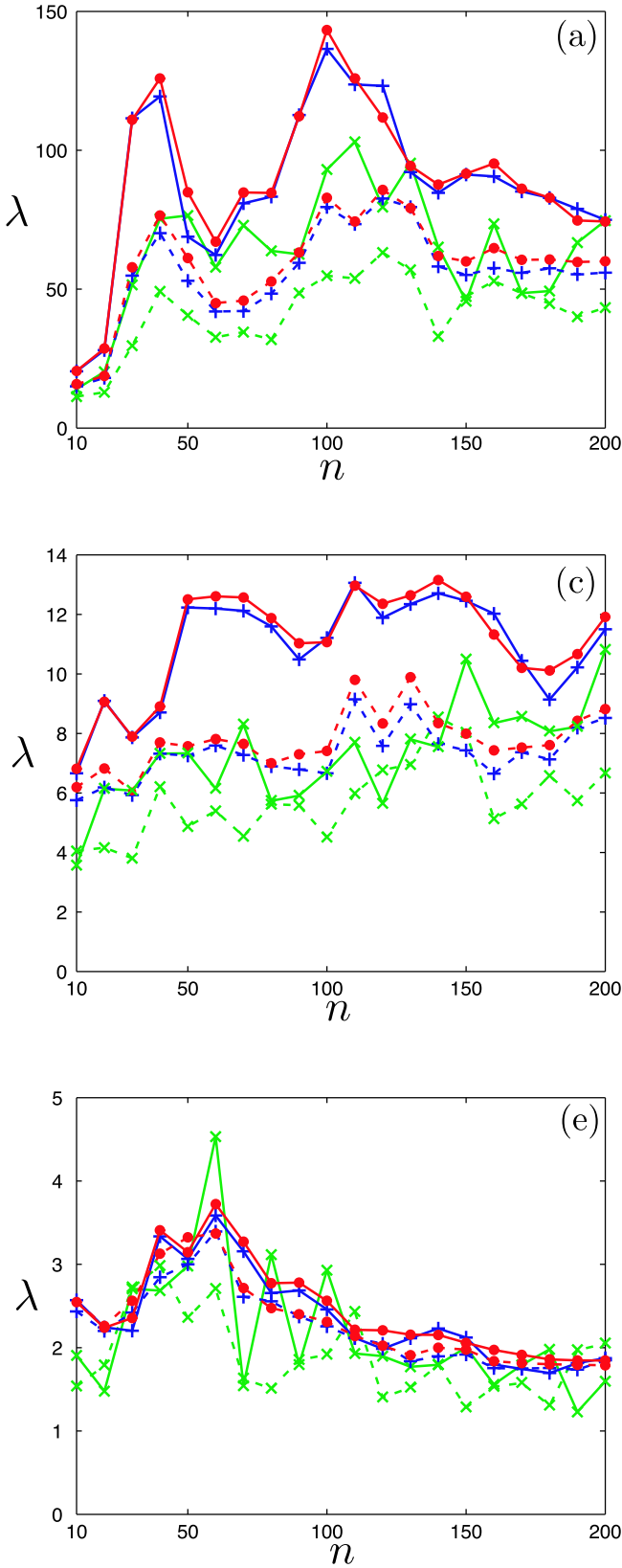

Fig. 7. Results of the asset growth rate $\lambda$ of our nonlinear portfolio model with the modified Sharpe ratio $S_{r}^{\prime}$ (solid lines). Each portfolio was rebalanced (a) everyday $(s=1)$, (b) every two days $(s=2)$, (c) every three days $(s=3)$, (d) every four days $(s=4)$, and (e) every five days $(s=5)$. Each dashed line means the same results as Figs. 6(b), (d), (f) based on the original Sharpe ratio $S_{r}$.

On the other hand, the results of using our nonlinear portfolio model are shown in Figs. 6(b), (d), and (f). We can see that each $\lambda$ is very improved even if stock-trading commissions and the trading-unit system are adopted. Especially, on the contrary, shorter-term rebalancing by using a smaller $s$ realizes better $\lambda$. Although we have to pay more stock-trading commissions caused by the larger frequency of portfolio rebalancing, the benefit of portfolio rebalancing by our nonlinear model to follow the rapid change of market trends is superior to the damage by rebalancing costs. Moreover, as shown in Fig. 4(c), short-term prediction works better for our nonlinear portfolio model although long-term predictions are difficult. This result supports the fact that stock-price movements follow a non-random walk [12], and therefore the active investment strategy is very useful.

Second, we examine the validity of the modified Sharpe ratio $S_{r}^{\prime}$ as an evaluation function for the portfolio optimization. Figure 7 shows the asset growth rate $\lambda$ given by our nonlinear portfolio model 
with the modified Sharpe ratio $S_{r}^{\prime}$ of Eq. (35). As a result, our modified Sharpe ratio $S_{r}^{\prime}$ can realize the higher asset growth rate $\lambda$ then the original Sharpe ratio $S_{r}$, and namely $S_{r}^{\prime}$ is very useful to optimize the allocation rates, considering stock-trading commissions for portfolio rebalancing.

Finally, we examine the relationship between the trading-unit system and the amount of the initial asset $M(0)$. As mentioned above, the trading-unit system makes the portfolio optimization problem more difficult, but it depends on the amount of $M(0)$. If we have enough amount of assets to consider the amount of each trading unit relatively small, we can ignore it because feasible solutions are not restricted. As results, in the case of $M(0)=10^{6}[¥]$, we can confirm the reduction of $\lambda$ because the best allocation rate $\left\{d_{i}^{*}\right\}$ was not a feasible solution due to the hurdle of the minimum trading amount. However, if we prepare more then $10^{7}[¥]$ as the initial asset $M(0)$, the investment performance $\lambda$ is converged, which means that $M(0)=10^{7}[¥]$ is large enough for portfolio management.

\section{Conclusion}

First, we modified the Markowitz mean-variance portfolio model to improve the prediction accuracy of future return rates by using nonlinear prediction model, because the original portfolio model can be considered to estimate the future return rate by a simple moving average of the recent historical data. However, because this modification caused a gap in the definition of a future risk, we redefine the risk from the viewpoint of the possibility of prediction errors similarly to the original Markowitz portfolio model, and then the future risk was estimated by historical prediction errors.

Next, we confirmed that we can select more predictable stocks to compose a multi-stock portfolio because there is the positive correlation between the unknown prediction accuracy and the known likelihood of nonlinear prediction. Moreover, we performed investment simulations with real stock data to demonstrate that our modified portfolio model can improve investment performance.

Especially, the main purpose of this study is to utilize our nonlinear portfolio model in real investment situations and to examine the best strategy for portfolio rebalancing, considering stock-trading commissions and the trading-unit system of minimum trading amount. Therefore, we also modified the Sharp ratio, which is an evaluation function of the portfolio optimization.

Through investment simulations, we confirmed as follows: as for the Markowitz portfolio model, short-term prediction of return rates does not work, and therefore portfolio rebalancing is ineffective. After all, stock-trading communions reduce the investment performance. On the other hand, as for our nonlinear portfolio model, short-term prediction works well and the portfolio rebalance is useful to follow real market trends, although we have to pay many commissions due to the high frequency of portfolio rebalancing. However, the benefit of portfolio rebalancing is superior to the damage by rebalancing costs. Moreover, we confirmed that our modified Sharpe ratio improves the investment performance because it can optimize allocation rates so as to reduce the rebalancing costs as much as possible. Finally, we examined the relationship between the trading-unit system and the amount of the initial asset. As a result, $10^{7}[¥]$ is enough large amount of the initial asset to prevent the minimum trading amount from restricting feasible solutions for our nonlinear portfolio model.

\section{References}

[1] H.M. Markowitz, "Portfolio selection," Journal of Finance, vol. 7, no. 1, pp. 77-91, 1952.

[2] E.F. Fama, "Efficient capital markets: A review of theory and empirical work," Journal of Finance, pp. 383-417, 1970.

[3] J.Y. Campbell, A.W. Lo, and A.C. Mackinlay, The Econometrics of Financial Markets, Princeton University Press, 1996.

[4] C. Gourieroux and J. Jasiak, Financial Econometrics, Problems, Models, and Methods., Princeton University Press, 2001.

[5] F. Black and R. Litterman, "Global portfolio optimization," Financial Analysts Journal, pp. 2843, 1992 .

[6] W.F. Sharpe, "Capital asset prices: A Theory of market equilibrium under conditions of risk," Journal of Finance, vol. 19, no. 3, pp. 425-442, 1964. 
[7] The historical data of the overnight unsecured call rate was obtained from The Tokyo Tanshi Co., Ltd., http://www.tokyotanshi.co.jp/past/index2.shtml, accessed on January 15, 2012.

[8] J.D. Farmer and J.J. Sidorowich, "Predicting chaotic time series," Phys. Rev. Lett., vol. 59, pp. 845-848, 1987.

[9] The historical stock data was bought from Pan Rolling Inc, http://www.panrolling.com/pansoft/data/, accessed on July 30, 2011.

[10] We used commission rate of Okasan Online Securities, http://www.okasan-online.co.jp/ cost_comparing/, accessed on November 30, 2012.

[11] B.G. Malkiel, A Random Walk Down Wall Street: The Time-Tested Strategy for Successful Investing, W.W. Norton \& Company, 2007.

[12] A.W. Lo and C.A. Mackinlay, A Non-Random Walk Down Wall Street, Princeton University Press, 2001. 